\title{
Connaissances Paysannes Et Prédisposition À Adopter Une Innovation En Agro-Alimentaire : Cas Du Décorticage Mécanique Et De La Fortification En Fer Du Sorgho Dans Le Nord-Bénin
}

\author{
Morest M. T. Agossadou \\ Sandrine S. Sêgla \\ Anselme A. Adégbidi \\ Université d'Abomey-Calavi, Faculté des Sciences Agronomiques, \\ Département d'Economie, Sociologie, Anthropologie et Communication \\ pour le Développement Rural \\ Polycarpe A. P. Kayodé \\ Université d'Abomey-Calavi, Faculté des Sciences Agronomiques, \\ Département de Nutrition et Sciences Alimentaires
}

Doi: 10.19044/esj.2018.v14n24p432 URL:http://dx.doi.org/10.19044/esj.2018.v14n24p432

\begin{abstract}
The consumption of meals prepared from unshelled sorghum derivatives is a factor favoring iron deficiency anemia among consumers. Mechanical dehulling and iron fortification of sorghum appears as a palliative solution. This study has a dual purpose. First of all, it is a question of assessing consumers' knowledge of sorghum meals with regard to anemia and its causes. Descriptive statistics have been used for this purpose. Then, it was discussed to analyze the determinants of the predisposition of these consumers to adopt mechanical dehulling and iron fortification of sorghum. To do this, the Logit econometric model was used. According to the results, anemia is caused by factors such as malaria, malnutrition, witchcraft, non-respect of fetishes, etc. In addition, households' propensity to adopt innovation is positively influenced by the income of the chef-cuisine, the perception that it has of its social status after the adoption of innovation and the compatibility of it with norms and values of the household. However, it is negatively influenced by the participation of the chef-cuisine in the experimentation phase and by the perceived complexity of the innovation.
\end{abstract}

Keywords: Anemia, sorghum, dehulling, iron fortification, predisposition to adopt, innovation. 


\section{Résumé}

La consommation de repas préparés à partir de dérivés de sorgho non décortiqués est un facteur favorisant l'anémie ferriprive au sein des consommateurs. Le décorticage mécanique et la fortification en fer du sorgho apparait comme solution palliative. La présente étude a un double objectif. Tout d'abord, il s'agit d'évaluer les connaissances des consommateurs de repas à base de sorgho relatives à l'anémie et à ses causes. La statistique descriptive a été utilisée à cet effet. Ensuite, il a été question d'analyser les déterminants de la prédisposition de ces consommateurs à adopter le décorticage mécanique et la fortification en fer du sorgho. Pour ce faire, le modèle économétrique Logit a été utilisé. D'après les résultats, l'anémie serait causée par des facteurs tels que le paludisme, la malnutrition, la sorcellerie, le non-respect des fétiches, etc. Par ailleurs, la prédisposition des ménages à adopter l'innovation est positivement influencée par le revenu de la chefcuisine, la perception qu'elle a de son statut social après l'adoption de l'innovation et la compatibilité de celle-ci avec les normes et les valeurs du ménage. Elle est cependant négativement influencée par la participation de la chef-cuisine à la phase d'expérimentation et par la complexité perçue de l'innovation.

Mots clés: Anémie, sorgho, décorticage, fortification en fer, prédisposition à adopter, innovation.

\section{Introduction}

La carence en fer touche 4 à 5 milliards de personnes soit 66 à $80 \%$ de la population mondiale (OMS, 2003). L'anémie quant à elle affecte environ 2 milliards de personnes (soit $30 \%$ de la population mondiale). D'après les chiffres de l'OMS, l'anémie en Afrique toucherait 45 millions d'enfants de moins de 5 ans, 58 millions de femmes en âge de procréer et 11 millions de femmes enceintes. En Afrique sub-saharienne, la carence en fer est la principale cause d'anémie. Cette forme d'anémie, causée par la carence en fer, est désignée sous le vocable d'anémie martiale (ou ferriprive). Les femmes en âge de procréer et les enfants constituent les groupes les plus vulnérables. Les conséquences de cette maladie sur ces groupes vulnérables sont énormes. L'anémie ferriprive provoque des dégâts irréversibles au niveau du cerveau, une diminution de la réponse immunitaire et donc une augmentation de la fréquence des infections chez les enfants en bas âge. Chez l'adulte, elle provoque de la fatigue et une capacité au travail réduite. Plus de naissances prématurées et d'enfants mort-nés sont les dégâts de ce mal chez la femme enceinte (Franziska, 2000). 
La carence en fer est principalement liée au fait que le fer alimentaire absorbé ne permet pas de couvrir les besoins élevés des populations à risques (Berger et Dillon, 2002 ; Franziska, 2000). Ceci est dû soit à la consommation d'aliments pauvres en fer, soit à la non ou faible disponibilité du fer contenu dans les aliments consommés. Pour faire face à ce problème, diverses stratégies existent comportant chacune des avantages et des inconvénients (Dillon, 2000). Il s'agit de la diversification alimentaire, de la supplémentation en fer, des mesures de santé publique et de l'enrichissement (ou fortification) en fer des aliments (Inwent, 2006 ; Alaoui, 2005 ; Berger et Dillon, 2002; Dillon, 2000). Dans les pays en développement, la fortification est de plus en plus, souvent, reconnue comme une approche efficace, à moyen et à long termes pour améliorer l'état en micronutriments de larges couches de la population (Inwent, 2006). Elle est considérée comme l'un des moyens les moins coûteux pour surmonter la malnutrition en micronutriments (Banque mondiale, 1994 ; Unicef, 1998). Elle consiste à ajouter du fer dans un aliment de consommation courante, afin d'augmenter le niveau de consommation de ce nutriment par la population (Inwent, 2006; Berger et Dillon, 2002). L'aliment qui transporte le nutriment est appelé véhicule ou vecteur.

Au Bénin, selon les statistiques de l'EDSB-III (2006), l'anémie ferriprive touche respectivement $78 \%$ et $61 \%$ d'enfants de 6 à 59 mois et de mères de 15 à 49 ans. Quatre vingt deux pour cent (82\%) des enfants anémiés se situent en milieu rural, contre $70 \%$ en milieu urbain. Chez les enfants, les taux d'anémie ferriprive les plus élevés se retrouvent dans les départements de l'Alibori $(89,7 \%)$, de la Donga $(88,1 \%)$ et les plus faibles dans les départements du Littoral $(60,3 \%)$ et du Borgou $(69,6 \%)$. Chez les mères, les taux d'anémie ferriprive sont les plus élevés dans les départements de l'Ouémé (75\%), l'Alibori (67\%), l'Atlantique (65\%), le Plateau (63\%) et le Mono $(61 \%)$. Les taux les plus faibles se retrouvent dans les autres départements et sont compris entre $50 \%$ et $57 \%$. Ces statistiques montrent que l'anémie ferriprive est un réel problème de santé publique au Bénin. Des actions concrètes doivent, de ce fait, être menées pour l'éradication de ce mal. Cet objectif ne peut être atteint sans l'identification des causes réelles de cette forme d'anémie dans ces milieux touchés. Il faut donc identifier le repas le plus consommé, voir sa composition chimique (notamment en fer) et évaluer la biodisponibilité de ce fer afin de savoir si l'anémie ferriprive, dans ces milieux, est liée à l'insuffisance de fer dans les repas consommés ou à la non disponibilité pour l'organisme du fer contenu dans les aliments consommés. Dans le premier cas, une fortification serait suffisante, mais dans le second, une amélioration de la biodisponibilité sera plus raisonnable.

Dans cette optique, une étude a été conduite dans le nord-Bénin par Kayodé et al (2005). Cette étude a révélé que le sorgho est une céréale qui occupe une place importante dans les habitudes alimentaires des populations 
concernées. Cette place importante qu’occupe cette céréale est imputable à la diversité d'aliments qui en sont issus, et à leur importance respective dans l'alimentation des ménages. Il s'agit des pâtes (dibou, sifanou, foura), des bouillies (koko, sorou, kamanguia) et des boissons (tchoukoutou, chakpalo). Selon ces mêmes auteurs, seulement 2 à $6 \%$ des $4 \mathrm{mg} / 100 \mathrm{~g}$ du fer contenu dans le sorgho est disponible aux consommateurs de ces types d'aliments. Ce faible niveau de disponibilité du fer est dû à son inhibition par les facteurs antinutritionnels (FAN), c'est-à-dire les tannins, le calcium/phosphore, les phytates et fibres et les oxalates, qui sont principalement localisés dans le péricarpe des graines (Kayodé et Denou, 2007). A partir de ce moment, la cause de l'anémie ferriprive dans le nord du Bénin n'est pas une insuffisance de fer dans les repas consommés, mais plutôt une faible disponibilité du fer contenu dans les aliments consommés.

Pour améliorer la biodisponibilité du fer contenu dans le sorgho, il convient, logiquement, de réduire, voir supprimer, les facteurs antinutritionnels qui y sont contenus et ce, par l'enlèvement du péricarpe des graines.

Il y a quelques décennies, les consommateurs du sorgho se livraient chaque jour à un travail manuel d'enlèvement du péricarpe et de pulvérisation des grains avant de pouvoir préparer le repas quotidien (Bassey et Schmidt, 1990). Les difficultés inhérentes à ces activités ont conduit ces consommateurs à désormais pulvériser directement les grains (de sorgho) avec l'apparition du moulin. A partir de ce fait, il ne serait plus raisonnable de proposer à ces consommateurs de sorgho d'enlever manuellement (ou traditionnellement) le péricarpe des grains. Il faut donc penser à une technologie pouvant jouer ce rôle. Mais l'enlèvement du péricarpe du sorgho, dans le but de réduire ou éliminer les facteurs antinutritionnels, n'est pas sans conséquences.

En effet, les $4 \mathrm{mg} / 100 \mathrm{~g}$ de fer contenu dans le grain de sorgho se localisent au même endroit que les facteurs antinutritionnels, i.e. dans le péricarpe. La réduction ou l'élimination des facteurs antinutritionnels par enlèvement du péricarpe entraine alors une réduction ou une élimination du fer contenu dans le sorgho. Pour ne pas revenir à la situation de départ où les consommateurs du sorgho entier présentent une carence en fer, un enrichissement des graines ou de la farine doit être effectué après l'enlèvement du péricarpe.

Ainsi, par le biais du projet intitulé ' Introduction of a mechanical dehuller and iron fortification in the traditional processing of sorghum in Benin to improve the iron status of rural consumers of porridge" initié par le Centre Régional de Nutrition et d'Alimentation Appliquées (CERNA) de la Faculté des Sciences Agronomiques de 1'Université d'Abomey-Calavi, une plate-forme de décorticage mécanique et de fortification en fer du sorgho est installée dans Thian, un village de la commune de Parakou, département du 
Borgou. Le décorticage ou l'enlèvement du péricarpe se fait de façon mécanique à l'aide d'un décortiqueur de type Engelbert et la fortification en fer est manuelle.

Le caractère nouveau de cette plate-forme et des services qu'elle offre fait d'elle une innovation. Une phase d'expérimentation de l'innovation a été effectuée entre octobre 2009 et juin 2010 dans le but d'évaluer son adaptation aux réalités alimentaires du village. Pour cette raison, vingt-quatre ménages contenant au moins un individu anémié ont été retenus pour décortiquer mécaniquement et fortifier en fer gratuitement le sorgho avant de préparer le repas. Avant le démarrage de la phase d'exécution proprement dite du projet, au cours de laquelle les services offerts par la plate-forme seront désormais payants et son accès sera donné à toute la population, quelques questions s'étaient posées : tous les ménages du village accepteront-ils décortiquer mécaniquement et fortifier leur sorgho avant de préparer le repas, dans le but d'améliorer leur statut en fer? quels sont les facteurs qui influencent cette décision de décortiquer, fortifier le sorgho? Le présent document vient répondre à ces différentes interrogations.

\section{Matériel et méthodes}

L'étude s'est déroulée dans le nord-Bénin, département du Borgou, commune de Parakou, et plus précisément dans le village Thian. Il s'agit du village retenu dans le cadre du projet, pour la phase d'expérimentation de l'innovation. C'est donc dans ledit village qu'est installée la plate-forme de décorticage mécanique et de fortification en fer du sorgho. Le décorticage mécanique se fait à l'aide d'un décortiqueur de type Engelbert fabriqué localement par le Centre de Recherche en Technologie Alimentaire et la fortification, à l'aide du NaFeEDTA fourni sous le nom commercial "Ferrazone", par la société Akzo Nobel. La figure 1 présente la carte du Département du Borgou mentionnant la commune d'étude, c'est-à-dire la commune de Parakou. 


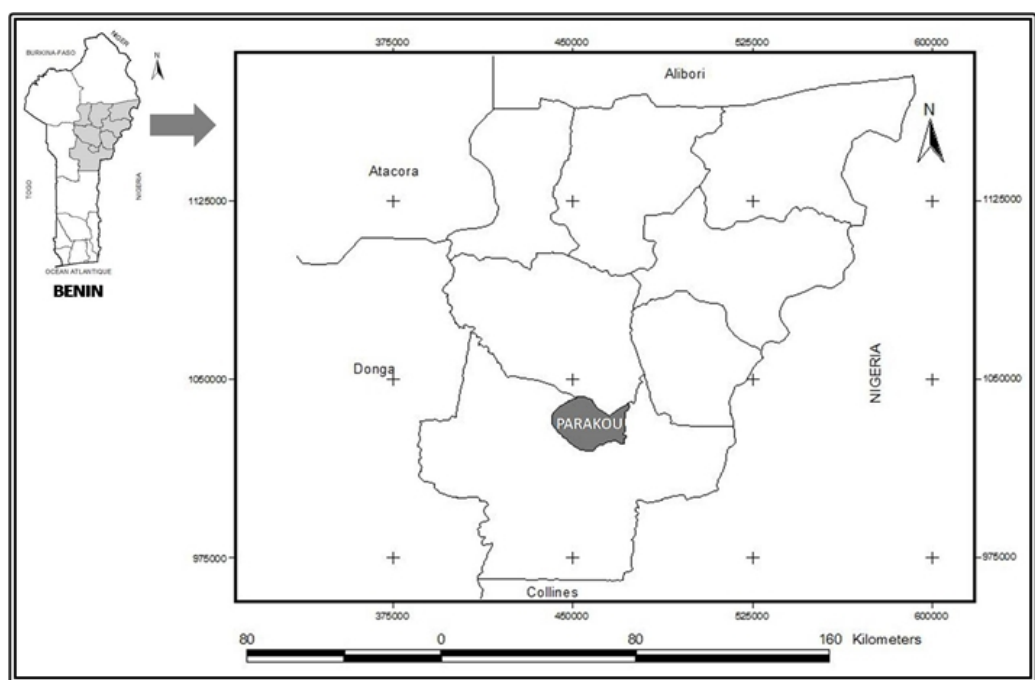

Figure 1. Carte du département du Borgou mentionnant la commune de Parakou.

Le mode d'échantillonnage aléatoire stratifié a été utilisé pour sélectionner 120 ménages "consommateurs" de dibou. Le critère de stratification est la participation ou non à la phase d'expérimentation de l'innovation ; nous avons alors deux strates : les ménages "consommateurs" de dibou retenus pour la phase d'expérimentation de l'innovation (strate 1) et les ménages "consommateurs" de dibou non retenus pour la phase d'expérimentation (strate 2). Les effectifs des strates 1 et 2 sont respectivement de 24 et 118 ménages.

En utilisant la méthode de proportionnalité, respectivement 20 et 100 ménages ont été aléatoirement tirés des strates 1 et 2 pour faire un total de 120 ménages comme échantillon de notre étude. Chaque ménage était représenté par la femme à charge de la préparation du dibou (chef-cuisine).

L'évaluation des connaissances des ménages consommateurs de dibou relatives à l'anémie s'est résumée à l'évaluation des causes de cette maladie selon les chefs-cuisine. Ainsi, une liste des causes possibles leur a été présentée. Il leur revenait de choisir la cause qu'elles jugent majeure. Des pourcentages ont été calculés pour analyser les choix des chefs-cuisines.

L'évaluation de la prédisposition des ménages à adopter l'innovation se résume à la mesure de l'influence des facteurs d'ordre socioéconomiques sur la disposition des chefs cuisine à adopter l'innovation. En d'autres termes, nous recherchons les variables socioéconomiques qui réduisent ou augmentent la probabilité de décider d'adopter le décorticage mécanique et la fortification en fer du sorgho tout en étant consciente que cette décision engendrerait des dépenses. A cet effet, les outils d'analyse les plus appropriés sont ceux de la régression. Mais, la difficulté majeure reste au niveau du choix du modèle de régression approprié. C'est d'abord la nature continue ou discontinue des 
variables qui déterminent le choix du modèle de régression (Maddala, 1983 ; Gourieroux, 1989). Ici, la variable dépendante est la prédisposition des ménages à adopter le décorticage mécanique et la fortification en fer du sorgho. Elle est dichotomique (ou binaire). Une variable dichotomique est une variable qui ne peut prendre que deux modalités exclusives l'une de l'autre, comme "Oui/Non" ou "Inférieur ou égal à/Strictement supérieur à". Dans le cas de notre étude, soit la chef-cuisine est prédisposée à adopter l'innovation soit elle ne l'est pas ; il faut donc recourir à des modèles économétriques appropriés.

Les modèles analytiques les plus largement utilisés dans ces types d'étude sont les modèles Probit (ou Normit), Logit et Tobit. Le modèle Tobit ou modèle de régression à variable dépendante limitée s'utilise lorsque nous avons des informations sur l'adoption ou non d'une innovation et mieux, si nous avons des informations sur le niveau d'utilisation de l'innovation (McDonald \& Moffit, 1980 ; Tobin, 1956). De plus, il fait appel à des calculs mathématiques assez complexes. Pour ces raisons, ce modèle d'analyse ne sera pas utilisé dans le cadre de cette étude. Les modèles Probit et Logit ont des caractéristiques proches (Amemiya, 1981). Il faut toutefois noter une différence majeure entre ces deux modèles. En effet, le modèle Logit se base sur la loi logistique de distribution de probabilité tandis que le modèle Probit se base sur la loi normale. Ces deux modèles aboutissent à des résultats similaires (Amemiya, ibid ; Maddala 1983). Dès lors, il n'y a pas de raison persuasive de choisir l'un plutôt que l'autre. Pratiquement, beaucoup de chercheurs adoptent le modèle Logit parce qu'il est mathématiquement plus simple (Gujarati 2004). Au regard de ce qui précède, le modèle Logit est retenu pour le test de cette première hypothèse. Etant donné que la variable dépendante dans notre cas ne peut prendre que deux valeurs (1 pour la prédisposition à adopter le décorticage mécanique et la fortification en fer du sorgho et 0 pour le cas contraire), nous avons utilisé le Logit binomial que nous avons estimé avec le logiciel statistique SPSS 16.0. Le modèle se spécifie comme suit :

Soit $P i$ la probabilité qu'associe le Logit à l'unité d'enquête $i$ :

$\mathrm{Pi}=\mathrm{F}\left(\mathrm{I}_{\mathrm{i}}\right)=\frac{1}{1+\mathrm{e}^{-\mathrm{I}_{\mathrm{i}}}}$

$I=\beta_{0}+\beta_{1} x_{i 1}+\beta_{2} x_{i 2}+\beta_{3} x_{i 3}+\cdots+\beta_{n} x_{i n}$

$\mathrm{I}_{\mathrm{i}}$ est un vecteur qui représente les caractéristiques de l'unité d'enquête, de son environnement et de l'objet de son choix. Les $\beta_{\mathrm{i}}$ représentent les coefficients des variables explicatives. Les $\mathrm{x}_{\text {in }}$ représentent les variables explicatives (ou régresseurs).

La décision de décortiquer, fortifier son sorgho et payer pour ces opérations intervient seulement lorsque l'effet combiné des facteurs atteint 
une valeur critique, à partir de laquelle l'individu choisit. En supposant que l'effet est mesuré par un indice non observable $\mathrm{I}_{\mathrm{m}}$ pour l'individu, et $\mathrm{I}_{\mathrm{m}}$, la valeur critique de l'indice à partir de laquelle il décide de décortiquer, fortifier son sorgho et payer pour ces opérations, on a : si $\mathrm{I}_{\mathrm{m}}$ est supérieur à $\mathrm{I}_{\mathrm{m}}$, alors l'individu choisit de décortiquer, fortifier son sorgho et payer pour ces opérations et la variable de choix Y prend la valeur 1 ; dans le cas contraire, Y est égale à 0 . Plus $I_{m}$ est supérieur à la valeur critique, plus la probabilité est forte que l'individu choisisse de décortiquer, fortifier son sorgho et payer pour ces opérations. Le modèle empirique peut s'écrire de la manière suivante :

$$
\begin{aligned}
\mathrm{P}_{\mathrm{i}}=\beta_{0}+\beta_{1} \text { AGE }+\beta_{2} \text { INSTRU }+\beta_{3} \text { REVENU }+\beta_{4} \text { ANEMIE }+\beta_{5} \text { MEPROD } \\
+\beta_{6} \text { QUANT }+\beta_{7} \text { APPP }+\beta_{8} \text { DISUNIT }+\beta_{9} \text { COMPLX } \\
+\beta_{10} \text { COMPAT }+\beta_{11} \text { STATU }+\beta_{12} \text { QUALMOUT }+\epsilon_{i}
\end{aligned}
$$

Les $\beta_{i}$ représentent les coefficients des variables explicatives et $\epsilon_{i}$ est le terme d'erreur.

AGE= Age de la chef-cuisine (en année)

INSTRU= Instruction de la "'chef cuisine" (Binaire ; $0=$ sans instruction, 1= avec instruction)

REVENU= Revenu de la chef-cuisine (en FCFA)

ANEMIE = Passé du ménage par rapport à l'anémie (Binaire ; $0=$ jamais d'anémié dans le ménage ; $1=$ au moins un membre du ménage déjà anémié)

MEPROD $=$ Production du sorgho par le ménage (Binaire ; $0=$ ménage non producteur; 1= ménage producteur)

QUANT= Quantité moyenne de sorgho consacrée à la consommation de dibou (en kg)

$\mathbf{A P P P}=$ Participation de la chef-cuisine à la phase d'expérimentation de l'innovation (Binaire ; $0=$ n'a pas appartenu; $1=$ a appartenu)

DISUNIT = Distance entre plate-forme et ménage de la chef-cuisine (en m)

COMPLX= Complexité perçue par la chef-cuisine, de la procédure de décorticage mécanique et de la fortification en fer du sorgho (Binaire ; $0=$ procédure non complexe $; 1=$ procédure complexe)

COMPAT = Compatibilité de la technologie avec les normes et valeurs du ménage de la chef-cuisine (Binaire ; $0=$ technologie non compatible ; $1=$ technologie compatible)

STATU = Statut social de la chef-cuisine après l'adoption de la technologie (Binaire ; $0=$ pas d'amélioration ; $1=$ amélioration)

QUALMOUT $=$ Qualité de la mouture au sein de l'unité $(0=$ mauvaise $; 1=$ acceptable ; $2=$ bonne)

\section{Résultats et discussions}

\section{Connaissances des chefs-cuisine sur les facteurs d'anémie}

La figure 2 présente les causes de l'anémie selon les "'chefs cuisine', enquêtées. 


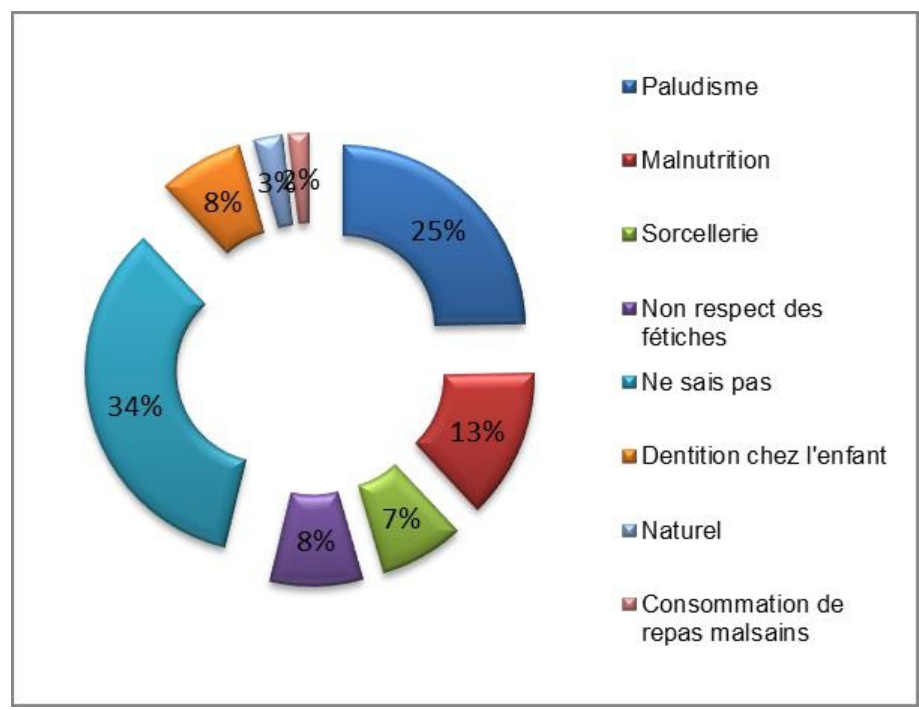

Figure 1: Les facteurs d'anémie selon les chef-cuisines.

Cette figure nous montre que les causes de l'anémie, selon les enquêtées, sont diverses. La plupart d'entre elles ignorent les réelles causes de l'anémie. Parmi celles qui lui connaissent au moins une cause, la majorité pense que le paludisme est le principal facteur favorisant l'anémie. Selon ces dernières, le fait pour un individu de s'exposer aux piqûres régulières de moustiques ou de s'exposer de façon régulière au soleil le rend vulnérable au paludisme. Et c'est le paludisme non ou mal traité qui conduit à l'amenuisement du sang dans l'organisme; d'où l'anémie. Pour les autres, les facteurs d'anémie sont : la malnutrition, la dentition chez l'enfant, le nonrespect des fétiches, la sorcellerie, les facteurs naturels et la consommation de repas malsains. L'encadré $\mathrm{N}^{\circ} 1$ rapporte les propos d'une chef-cuisine pour qui l'anémie est causée par les fétiches.

ENCADRE N'1
Aucun membre de mon ménage n'a jamais souffert de cette maladie. Il y a déjà des années,
j'ai vu une femme très riche souffrir de ça. Les gens l'on amenée à l'hôpital et malgré tous
les soins qu'elle y a reçus, la maladie n'a pas cessé ; elle manquait cruellement de sang.
Cela a fini par inquiéter ses proches qui ont commencé par chercher dans la tradition, les
causes du mal. Ils ont découvert que c'est un fétiche qui est à la base de la maladie.
En fait, la dame a vendu son âme au diable, à travers ce fétiche, pour que ces activités
marchent et fleurissent très bien, afin d'avoir beaucoup d'argent. Naturellement, il y a des
sacrifices qu'elle doit faire périodiquement. Il s'agissait de donner du sang de mouton au
fétiche suivant une périodicité bien précise. Et il paraîtrait que c'est pour le fait qu'elle ait
manqué de faire ce sacrifice une fois, que le fétiche s'est mis en colère contre elle. Cette
colère s'est manifestée par l'extinction de son sang en lieu et place du sang du mouton.
C'est le seul cas que j'ai connu; et c'est pourquoi je dis que c'est le non-respect des
promesses faites à un fétiche qui est à la base de l'anémie.

${ }^{1}$ : Le nom du fétiche est connue mais option a été faite de le taire pour éviter d'éventuelles frustrations. 


\section{Prédisposition des ménages consommateurs de dibou à adopter le décorticage mécanique et la fortification en fer du sorgho}

Le tableau 1 présente les résultats de la régression logistique.

Tableau 1 : Résultats de la régression logistique binomiale.

\begin{tabular}{|c|c|c|c|c|c|}
\hline $\begin{array}{l}\text { Variables } \\
\text { explicatives }\end{array}$ & $\begin{array}{l}\text { Définition de la } \\
\text { variable }\end{array}$ & $\begin{array}{l}\text { Signes } \\
\text { Attendus }\end{array}$ & Coefficients & Probabilités & $\begin{array}{l}\text { Degré de } \\
\text { signification }\end{array}$ \\
\hline Constante & 1 & 1 & $-8,280$ & 0,0001 & **** \\
\hline AGE & Age de la "chef cuisine" & - & $-0,100$ & 0,171 & ns \\
\hline INSTRU & $\begin{array}{l}\text { Instruction de "chef } \\
\text { cuisine" }\end{array}$ & + & $-1,958$ & 0,315 & ns \\
\hline REVENU & $\begin{array}{l}\text { Revenu de la "chef } \\
\text { cuisine". }\end{array}$ & + & 0,0001 & 0,044 & $* *$ \\
\hline ANEMIE & $\begin{array}{l}\text { Passé du ménage par } \\
\text { rapport à l'anémie }\end{array}$ & + & 0,487 & 0,719 & ns \\
\hline MEPROD & $\begin{array}{l}\text { Production du sorgho par } \\
\text { le ménage }\end{array}$ & + & $-1,975$ & 0,207 & ns \\
\hline QUANT & $\begin{array}{l}\text { Quantité moyenne de } \\
\text { sorgho consacrée à la } \\
\text { consommation de dibou }\end{array}$ & + & $-0,272$ & 0,154 & ns \\
\hline APPP & $\begin{array}{l}\text { Participation de la 'chef } \\
\text { cuisine'" à la phase } \\
\text { d'expérimentation de } \\
\text { l'innovation. }\end{array}$ & + & $-3,974$ & 0,022 & ** \\
\hline DISUNIT & $\begin{array}{l}\text { Distance entre plate-forme } \\
\text { et ménage de la "chef } \\
\text { cuisine", }\end{array}$ & - & 0,003 & 0,273 & ns \\
\hline COMPLX & $\begin{array}{l}\text { Complexité perçue par la } \\
\text { "chef cuisine" de la } \\
\text { procédure de décorticage } \\
\text { mécanique et de la } \\
\text { fortification en fer du } \\
\text { sorgho }\end{array}$ & - & $-5,801$ & 0,024 & ** \\
\hline COMPAT & $\begin{array}{l}\text { Compatibilité de la } \\
\text { technologie avec les } \\
\text { normes et valeurs du } \\
\text { ménage de la "chef } \\
\text { cuisine". }\end{array}$ & + & 8,481 & 0,020 & $* *$ \\
\hline STATU & $\begin{array}{l}\text { statut social de la 'chef } \\
\text { cuisine"' après l'adoption } \\
\text { de la technologie. }\end{array}$ & + & 5,616 & 0,029 & $* *$ \\
\hline QUALMOUT & $\begin{array}{l}\text { Qualité de la mouture au } \\
\text { sein de l'unité }\end{array}$ & + & 1,093 & 0,227 & ns \\
\hline \multicolumn{6}{|c|}{ Nombre d'observations $=120 ;$ Nombre de prédisposées $=90 ;$ Nombre de non prédisposées $=30$. } \\
\hline \multicolumn{6}{|c|}{-2 Log vraisemblance $=28,134$} \\
\hline \multicolumn{6}{|c|}{ Khi-deux $=58,853$} \\
\hline \multicolumn{6}{|c|}{ Significativité du modèle: $0,0001 * * *$} \\
\hline \multicolumn{6}{|c|}{ Pouvoir de prédiction: $54,4 \%$ (Cox et Snell); 79,2\% (Nagelkerke) } \\
\hline$* * *=$ Signif & à $1 \% ; * *=$ Significatif à & $n s=N$ & snificatif. & & \\
\hline
\end{tabular}

Variable dépendante : Prédisposition à adopter le décorticage et la fortification du sorgho. 
Le ratio de vraisemblance s'est révélé significatif à $1 \%$ après le test de khi-deux. Le modèle est donc globalement significatif à $1 \%$. Les résultats du modèle (notamment les signes des coefficients) peuvent être valablement pris en compte. Les variations de la variable indépendante sont alors expliquées par les variables dépendantes de manière acceptable. Le pseudo- $\mathrm{R}^{2}$ de Nagelkerke est égal à 0,792 . On peut donc, à partir du modèle, faire des prévisions sur les modalités de la variable dépendante, connaissant celles des variables indépendantes avec une probabilité allant à 79,2\% d'avoir une prédiction juste. Cinq (5) facteurs influencent significativement la prédisposition des ménages consommateurs de dibou à adopter le décorticage mécanique et la fortification en fer du sorgho. Il s'agit du revenu de la chefcuisine (REVENU), de sa participation à la phase d'expérimentation de l'innovation (APPP), la complexité perçue de la procédure de décorticage mécanique et de la fortification en fer du sorgho (COMPLX), la compatibilité de l'innovation avec les normes et valeurs du ménage de la chef-cuisine (COMPAT) et la perception de la chef-cuisine de son statut social après l'adoption de la technologie (STATU).

\section{$\rightarrow$ Revenu de la chef-cuisine}

Il s'agit du revenu des femmes à charge de la préparation de la pâte du sorgho dans les ménages enquêtés. Dans le modèle, le signe du coefficient de cette variable est le signe plus (+) ; il correspond au signe attendu. Il indique que la décision d'adopter le décorticage mécanique et la fortification en fer du sorgho est positivement influencée par le revenu de la chef-cuisine. En d'autres termes, plus le revenu de la chef-cuisine est élevé, plus grande est sa probabilité de décider d'adopter l'innovation. Ce résultat s'explique par le fait que les chef-cuisines qui ont un revenu élevé se sentent en mesure de faire face aux coûts liés à l'adoption de l'innovation. En effet, avant l'obtention du sorgho décortiqué et fortifié, diverses opérations sont effectuées par le minotier et l'agent qui assure la fortification. Il s'agit dans cet ordre de la pesée du sorgho à l'aide d'une balance mécanique, de l'humidification du sorgho, du décorticage proprement dit du sorgho à l'aide du décortiqueur, de la pesée du sorgho décortiqué à l'aide de la même balance mécanique, de la pesée de la quantité de fer nécessaire à la fortification du sorgho décortiqué à l'aide d'une balance électrique, de la fortification proprement dite du sorgho décortiqué. La chef-cuisine ayant pris conscience de ce que toutes ces opérations ont un coût, fait référence à son niveau de revenu et se prononce sur sa décision d'adopter ou de rejeter l'innovation. Il va s'en dire que la femme paysanne contribue financièrement à nourrir son ménage en plus du travail physique des opérations de cuisson. 


\section{$\rightarrow$ Participation de la chef-cuisine à la phase d'expérimentation de l'innovation}

Cette variable désigne la participation ou non de la chef-cuisine à la phase pilote du projet qui a conduit à l'installation de l'unité de décorticage et de fortification. Les résultats obtenus à partir de la régression logistique montrent que cette variable influence négativement la décision d'adopter le décorticage mécanique et la fortification en fer du sorgho. La participation à la phase pilote du projet est donc négativement corrélée à la disposition de la chef-cuisine à adopter le décorticage mécanique et la fortification en fer du sorgho. Autrement dit, lorsqu'on va des chef-cuisine n'ayant pas appartenu à la phase pilote du projet à celles ayant appartenu à la phase pilote, nous avons une diminution du nombre des prédisposées. Cet effet négatif de cette variable sur la prédisposition de la chef-cuisine à adopter l'innovation est contraire à celui que nous avions prédit. Selon Evenson (1992) et Ogunlana (2003), le contact de l'enquêté avec la "vulgarisation" devrait faciliter l'accès à l'information et favoriserait l'adoption des innovations. Mais, l'effet contraire observé au cours de notre étude pourrait s'expliquer par les multiples plaintes de ces femmes qui ont appartenu à la phase pilote du projet, et donc expérimentées la technologie. En effet, pendant les huit mois d'expérimentation de la technologie par les femmes sélectionnées, elles ont relevé plusieurs insuffisances. Il s'agit de :

$\checkmark$ la non-permanence de l'agent qui effectue la fortification : en fait, pour le compte du projet, un agent a été recruté pour assurer la fortification du sorgho, après le décorticage par le minotier. Cet agent, dans une journée, n'arrive dans le village pour faire son travail, qu'en fin d'après-midi (17 heures). A son arrivée, dès qu'il finit de fortifier le sorgho déjà décortiqué, il rebrousse chemin. Alors, les femmes qui amènent du sorgho dans l'unité en son absence et qui sont dans un besoin pressant de farine pour préparer la pâte ne peuvent pas voir leur sorgho fortifié. Cette situation n'est pas du goût des chef-cuisines qui ont déjà expérimenté la technologie.

$\checkmark \quad$ l'impossibilité de conserver la farine sur une longue durée. Selon les chef-cuisines, la farine qu'elles obtenaient avec le sorgho non décortiqué se conservait pendant plus de deux semaines, alors que celle améliorée (issus de sorgho décortiqué et fortifié) ne se conserve pas pendant plus de 3 ou 5 jours. Ceci est certainement dû, selon ces chef-cuisine à l'humidification que subit le sorgho avant son décorticage. Cette humidification augmente le taux d'humidité de la farine favorisant ainsi l'activité des micro-organismes, et conduisant à son altération précoce. Cette situation n'avantage guère les chefcuisines.

$\checkmark$ la diminution de la quantité de farine après décorticage : même si les chef-cuisines ont reconnu qu'il ne peut en être autrement, elles ont tout de même mentionné cet état de chose, lors des discussions que nous avons 
menées avec elles. Cela pourrait les amener à préférer moudre leur sorgho sans forcément passer par le décorticage.

$\rightarrow$ Complexité perçue de la procédure de décorticage mécanique et de la fortification en fer du sorgho

Il s'agit de la perception de la chef-cuisine sur la complexité ou non de la procédure de décorticage et de fortification. Cette perception était évaluée à l'aide de deux critères: le niveau de facilité de compréhension de la procédure de décorticage et de fortification et l'idée que la chef-cuisine se fait du temps que prendra ces opérations. Ainsi, la procédure est qualifiée de complexe lorsque la chef-cuisine affirme qu'elle est difficile à comprendre, ou lorsque la chef-cuisine juge la procédure trop longue. A travers le modèle, nous voyons que cette variable influence négativement la prédisposition des chef-cuisines à adopter l'innovation. Cette influence négative, conforme à nos prévisions, indique que la probabilité de rejet de l'innovation augmente lorsque l'enquêtée juge la procédure de décorticage et de fortification complexe. Un résultat semblable a été obtenu par Ogunlana (2003) lorsqu'elle conduisait son étude sur les comportements d'adoption de la culture en couloir par les agricultrices du Nigéria. Cet auteur a montré que la complexité, définie dans le cadre de son étude comme le degré auquel une innovation est perçue comme étant difficile à comprendre et à utiliser, influençait négativement l'adoption de la culture en couloir.

$\rightarrow$ Compatibilité de la technologie avec les normes et valeurs du ménage de la 'chef cuisine"'

Cette variable a été introduite dans le modèle de régression logistique pour voir l'influence des normes et valeurs du groupe social qu'est le ménage auquel la chef-cuisine appartient, sur la prédisposition à adopter l'innovation. Ces normes et valeurs peuvent être liées à la tradition, à la religion, aux croyances, etc. En exemple, pour certaines chef-cuisines, décortiquer le sorgho est synonyme de "dépigmenter" le sorgho ; la dépigmentation étant un acte contraire à la volonté de Dieu. Pour d'autres, l'enlèvement du fer "naturel" contenu dans le péricarpe du sorgho et son remplacement par du fer "non naturel' (ou 'fabriqué par des humains") constitue un acte qui va en l'encontre des 'lois de la nature'. De plus, pour ces dernières, le fer 'fabriqué par les humains"' serait d'une qualité moins bonne que celui 'naturel'. Les résultats du modèle révèlent un effet positif de cette variable sur la prédisposition des "chefs cuisine" à adopter le décorticage mécanique et la fortification en fer du sorgho. Cette variable est donc positivement corrélée avec la prédisposition à adopter l'innovation. Cet effet positif est celui qui est attendu. Il indique que la probabilité d'acceptation de l'innovation augmente lorsqu'elle est compatible avec les normes et valeurs de la chef-cuisine. 
$\rightarrow$ Statut social de la 'chef cuisine"'après adoption de l'innovation

Il s'agit ici de l'idée que la chef-cuisine se fait de son statut social, une fois qu'elle aura adopté l'innovation. Dans le modèle, le signe du coefficient de cette variable est le signe plus (+) ; il correspond au signe attendu. Il indique que la décision d'adopter le décorticage mécanique et la fortification en fer du sorgho est positivement influencée par le statut social de la chef-cuisine. Autrement dit, la probabilité d'acceptation de l'innovation augmente lorsque la chef-cuisine est convaincue qu'elle améliorera son statut dans le village. Le statut social de la chef-cuisine est amélioré, lorsque celle-ci est mieux 'regardée" que par le passé. En effet, l'adoption du décorticage mécanique et de la fortification en fer du sorgho est source de prestige car, d'une manière ou d'une autre, elle implique des charges financières supplémentaires. Une femme qui adopte cette innovation est une femme qui est en mesure de dépenser plus d'argent que par le passé. Etre capable de sortir de son portefeuille plus d'argent que par le passé, fait de cette femme et de son époux, des personnes qu'on peut qualifier d'aisées. En dehors de cela, un ménage qui accepte décortiquer et fortifier chaque fois le sorgho avant de le moudre, pourra être vu comme un ménage exemplaire, car ayant le souci de contribuer à l'éradication d'une maladie. Tout ceci contribuerait à l'amélioration du statut social des chef-cuisines qui adopteraient l'innovation. Celles qui sont convaincues de cela ont une grande probabilité d'adoption du décorticage et de la fortification. Un résultat similaire a été trouvé par Ogunlana (2003).

\section{Conclusion}

Cette étude a montré que les connaissances des populations rurales, relatives aux facteurs responsables de l'anémie sont multiples et multiformes. Ces facteurs sont entre autres le paludisme, la malnutrition, la sorcellerie, le non-respect des fétiches, etc. L'étude a également révélé que le revenu de la chef-cuisine, la compatibilité de l'innovation avec ses normes et valeurs et la perception qu'elle a de son statut social après l'adoption de l'innovation sont les facteurs qui contribuent favorablement et significativement à l'adoption du décorticage mécanique et de la fortification en fer du sorgho. Par contre, la participation de la chef-cuisine à la phase d'expérimentation de l'innovation et sa perception de la complexité de la procédure de décorticage mécanique et de la fortification en fer du sorgho constituent des éléments qui peuvent inhiber l'adoption de l'innovation.

Dans l'optique donc d'une large adoption du décorticage mécanique et de la fortification en fer du sorgho, il importe d'une part, de renforcer les forces d'impulsion et d'autre part, d'éliminer les forces d'inhibition. L'élimination de ces forces d'inhibition passera par : 
$\nabla$ l'amélioration du pouvoir de conservation de la farine obtenue à partir du sorgho décortiqué et fortifié ;

$\nabla$ l'allègement du procédé de décorticage mécanique et de fortification en fer du sorgho ;

$\nabla$ le renforcement des capacités du meunier afin qu'il soit en mesure d'assurer la fortification du sorgho décortiqué. Cela pallierait le problème de non-permanence de l'agent qui assure la fortification ;

$\nabla$ l'amélioration du rendement du décortiqueur afin de pallier le problème de la réduction de la farine après le décorticage ; et

$\nabla$ l'initiation de campagnes d'information, d'éducation et de communication afin de lever les contraintes socioculturelles liées à l'acceptation de l'innovation.

\section{References:}

1. Amemiya T, 1981. Qualitative response model : a survey. Journal of Economic Literature 19, 1483-1536.

2. Evenson RE, 1992. Research and extension in agricultural development. In: Occasional papers, $\mathrm{n}^{\circ}$ 25. International Center For Economic Growth, University of Yale Connecticut.

3. Gourieroux C, 1989. Econométrie des variables qualitatives. 2nd edn, Economica, Paris.

4. Gujarati DN, 2004. Econométrie. Traduction de la 4è édition américaine par B. Bernier.

5. Kayodé APP, Adégbidi A, Linnemann AR, Nout MJR, Hounhouigan JD, 2007. Perception par les consommateurs de la qualité des variétés de sorgho paysannes en usage au Bénin et de leurs produits dérivés. Ecology of Food and Nutrition 44, 271-294.

6. Maddala GS, 1983. Limited dependent and qualitative variables. In: econometrics. Cambridge University Press, Cambridge.

7. McDonald J F, MOFFIT R A, 1980. The uses of Tobit analysis. Rev. Econ. Stat. $62: 318321$.

8. Ogunlana EA, 2003. The technology adoption behavior of women farmers: The case of alley farming in Nigeria. Renewable Agriculture and Food Systems 19; 57-65.

9. TOBIN J, 1956. Estimation of relationship for limited dependent variables. Econometrica, $26: 24-36$. 